\title{
Determinación de densidades urbanas sostenibles en base a metodología relativa al acceso solar: caso área metropolitana de Mendoza, Argentina
}

\author{
Determination of sustainable urban densities based on a methodology related to solar access: study case of the \\ metropolitan area of Mendoza, Argentina
}

Jimena Gómez Piovano* y Alejandro Mesa*

Recibido: 29 de noviembre de 2016

Aceptado: 12 de junio de 2017

\section{Resumen}

Uno de los retos actuales a nivel de diseño y planificación urbana consiste en la trasformación de las urbes actuales en modelos de ciudades que contemplen el cuidado del medio ambiente y la mejora de la calidad de vida de los habitantes. Los estudios realizados denotan que los conglomerados de baja densidad poblacional son los más contaminantes (Cepeda y Mardaras, 2004; Norman, Maclean, Asce \& Kennedy, 2006) en consecuencia una de las mejoras urbanas a fomentar es la densificación de las ciudades. No obstante, los niveles poblacionales no son únicos y estándares, sino que deben encontrarse en relación con las características propias de cada sector. Este trabajo tiene como objetivo principal desarrollar una metodología de cálculo que permita determinar niveles poblacionales máximos contemplando los requerimientos bioclimáticos de la ciudad y garantizando el acceso al sol más igualitario para todos los habitantes de la urbe. La misma es aplicada en el Área Metropolita de Mendoza y denota que las dimensiones de las manzanas y el ancho de las calles limitan la capacidad constructiva del sector $y$, en consecuencia, la cantidad de personas albergable. Los resultados obtenidos dan cuenta de las densidades poblaciones máximas para los distintos sectores, las que varían entre los 49 y 400 habitantes por hectárea, siendo las manzanas angostas con calles estrechas las de menor capacidad.

Palabras clave: acceso al sol, densidad poblacional, indicadores urbanos, urbanismo bioclimático.

\begin{abstract}
One of the current challenges in terms of design and urban planning involves the transformation of existing cities in models of cities that take care of the environment and improve the quality of life of the inhabitants. Studies indicate that clusters of low population density are the most polluting, (Cepeda y Mardaras, 2004; Norman, Maclean, Asce \& Kennedy, 2006) consequently urban densification is one improvement to promote. However, population levels are not unique and standard, but must be found in relation to the characteristics of each urban area. This work has as main objective to develop a methodology of calculation to determine maximum population levels considering the bioclimatic requirements of the city and ensuring more equitable access to sunlight for all the inhabitants of the city. The same is applied in the Metropolitan Area of Mendoza and denotes that the dimensions of the blocks and the width of the streets limit the constructive capacity of the sector and consequently the number of people accommodating. The results obtained indicate that the maximum population densities for the different sectors vary between 49 and 400 inhabitants per hectare, with narrow blocks with narrow streets being the ones with the lowest capacity.
\end{abstract}

Keywords: bioclimatic urban planning, population density, sun accessibility, urban indicators.

\footnotetext{
*Instituto de Ambiente, Hábitat y Energía, Centro Científico Tecnológico del Consejo Nacional de Investigaciones Científicas y Técnicas (CONICET), Mendoza, Argentina. Contacto: jpiovano@mendoza-conicet.gob.ar

Se agradece al Consejo Nacional de Investigaciones Científicas y Técnicas (CONICET), quien financió esta investigación a través de su programa de becas de doctorado.

Cómo citar: Gómez Piovano, J. y Mesa, A. (2017). Determinación de densidades urbanas sostenibles en base a metodología relativa al acceso solar: caso área metropolitana de Mendoza, Argentina. Revista de Urbanismo, 36, $131-145$. http://dx.doi.org/10.5354/0717-5051.2017.44367
} 


\section{Introducción}

Desde la publicación del Informe de Naciones Unidas Our Common Future, también conocido como Informe de Brundtland, en 1987, el concepto de "desarrollo sostenible" ha sido contextualizado en torno a las distintas actividades o temáticas. Existe gran cantidad de estudios, teorías y normativas que evidencian la importancia de transformar a las urbes actuales en modelos de ciudades que contemplen el cuidado del medio ambiente y la mejora de la calidad de vida de los habitantes. Las primeras declaraciones surgen en 1990 con la publicación del Libro Verde del Medio Ambiente Urbano, donde la Comisión Europea puso de manifiesto la importancia de incluir la conciencia ecológica en la planificación de las ciudades (López y Relea, 2002; Valenzuela, 2009). Posteriormente, la Organización de Naciones Unidas:

(...) catapultó a la escala global el concepto de desarrollo urbano sostenible desde la plataforma de la Conferencia de Río sobre Medio Ambiente y Desarrollo (1992) integrándolo en el Plan de Acción para el Siglo 21 y en su prolongación, la Iniciativa Agenda Local 21 (Valenzuela, 2009, p. 408).

Desde esas primeras publicaciones hasta hoy en día el desarrollo urbano sostenible ha sido un tema prioritario de investigación y acción, siendo el principal objetivo convertir a nuestras ciudades contaminantes en espacios que se adapten tanto a quienes las habitan como a la naturaleza (Hernández, 2009).

Diversos autores abordan el desarrollo urbano sostenible desde distintas perspectivas, pero todos concuerdan en que las principales características son: la compacidad, la eficiencia, la cohesión social, la complejidad, la caminabilidad, la conectividad, el espacio público y la equidad (Gaja, 2008; González, 2005; López y Relea, 2002; Marín, 2012; Rueda, 2011).

El modelo de ocupación del suelo compacto es uno de los pilares fundamentales en el diseño de ciudades más sostenibles. Dicho patrón supone mejores comportamientos que los modelos dispersos o difusos en los distintos ámbitos que integran la ciudad. Primeramente, la compacidad urbana implica un menor consumo de suelos, conservando así mayor cantidad de suelo agrícola y natural, "disminuyendo, así, la presión de los sistemas urbanos sobre los sistemas de apoyo"
(Rueda, 2011 p. 25) y a su vez, promueve mayor densidad poblacional. La mayor concentración de personas sobre un sector favorece la diversidad de usos en un barrio o en un territorio concreto, es decir, la convivencia entre la residencia, los servicios, las actividades económicas, los equipamientos, etc. De esta manera proporciona el contexto adecuado para el aumento de intercambios de bienes y servicios (Rueda, 2011).

A nivel de eficiencia, un nivel de densidad poblacional medio posibilita la coexistencia de servicios y equipamientos urbanos necesarios para los pobladores en el entorno inmediato, disminuyendo la necesidad de uso de transporte a motor y las emisiones de $\mathrm{CO}_{2}$ que ello implica. Dichos beneficios no solo repercuten a nivel ambiental, sino que la disminución del número de automóviles circulando mejora la calidad del espacio público, debido a que gran parte de las vías de movilidad se encuentran hoy saturadas por el tránsito privado.

Asimismo, la agrupación de edificios de fachada continua disminuye la superficie de envolvente expuesta, reduciendo así los requerimientos calefacción en invierno y refrigeración en verano (Mesa y De Rosas, 2001). Esto se debe a que se reducen las superficies de intercambio térmico. De la misma manera, disminuye la cantidad de materiales de construcción. La densificación, en relación con la infraestructura, disminuye las extensiones de las redes de servicios, lo cual se traduce en menores costos y aumento de la eficiencia (Mesa y de Rosas, 2001).

En relación con ello, un grupo de investigadores canadienses encabezados por Jonathan Norman (2006) cuantificó las diferencias de consumo energético de dos modelos urbanos, uno difuso de baja densidad (57 hab./ha) y el otro compacto de mayor densidad (270 hab./ha) ubicados en la Ciudad de Toronto (Latitud $\left.43^{\circ} 42^{\prime} 00^{\prime \prime} \mathrm{N}\right)$. Sobre la base del análisis realizado determinaron que el sector más denso consume 37\% menos de energía en construcción y $45 \%$ menos en relación con la operatividad edilicia. Como consecuencia de la menor distancia y la disminución de viajes, se reduce el consumo energético del transporte privado entre un $73 \%$ y un $70 \%$ menos en los recorridos de transporte público respecto del sector de baja densidad.

De este modo el modelo de ciudad difusa se considera insostenible, ya que la huella creciente de ocupación por el suelo urbano y la red de movilidad 
horizontal invade tierras de alto valor tanto ecológico como productivo, generando mayores consumos energéticos y, por lo tanto, mayor contaminación.

No obstante, así como la baja densidad es insostenible, el exceso de densidad puede producir problemas ambientales. Gran cantidad de autores exponen que los niveles de densidad apropiados no son rígidos, sino que varían dependiendo de las características propias de cada ciudad. No obstante, se establecen rangos óptimos, que van desde 120 habitantes por hectárea a 350 (Higueras, 2009; Marín, 2012; Rueda, 2011).

En la búsqueda de las mejoras del nivel de sostenibilidad de las ciudades actuales es fundamental incluir:

(...) criterios de economía energética, y de aprovechamiento de los recursos medioambientales de cada localidad, para que se equilibre el diseño urbano con las variables climáticas, topográficas y específicas de cada municipio y así conseguir una adecuación en todos los aspectos (Higueras, 1998, p. 5).

Consecuentemente, la incorporación de estrategias bioclimáticas en el diseño es fundamental para mejorar la eficiencia de la ciudad a nivel energético.

Actualmente, el mayor consumo energético a nivel edilicio se produce para alcanzar estándares de confort en los espacios interiores. A tal fin, mayormente se utilizan energías no renovables en calefacción, refrigeración, ventilación e iluminación (Cárdenas y Uribe, 2012). La incorporación de estrategias de diseño bioclimático en los edificios posibilita la reducción del uso de energías contaminantes mediante el acondicionamiento natural de los espacios.

El sol es la principal fuente de energía renovable en ciudades como Mendoza, por lo que pensar en ciudades más eficientes sin la explotación del recurso solar resulta inviable. El potencial calórico del sol se utiliza para la climatización natural de los espacios interiores, así como también para mejorar el confort de los espacios abiertos en los periodos fríos. La energía solar puede ser canalizada a través de sistemas pasivos o activos. EI acondicionamiento pasivo de los ambientes es concebido desde el diseño de los edificios y requiere que se garanticen a nivel urbano ciertos niveles de asoleamiento. Los sistemas activos son aquellos capaces de generar energía.

Las potencialidades de aprovechamiento del sol no solo están relacionadas con la generación de energía o la calefacción, sino que por un lado también posibilita la iluminación natural de los espacios y, por otro, en las épocas de calor, la radiación permite la presencia y el crecimiento de vegetación. La evapotranspiración de la misma durante los períodos cálidos contribuye a refrescar el medio ambiente construido, disminuyendo la necesidad de energía para refrigeración (Cummins \& Jackson, 2001; Ruiz, Correa y Cantón, 2012).

El urbanismo bioclimático tiene por objetivo mejorar los niveles de confort, tanto térmico como lumínico, de los habitantes de una ciudad, haciendo uso de los recursos naturales disponibles y minimizando el uso de energías no renovables. En definitiva, debe permitir el máximo aprovechamiento de los recursos disponibles, de tal modo que las construcciones puedan incorporar estrategias de climatización pasivas y sistemas de generación energética, mitigar los efectos microclimáticos negativos de las ciudades, así como también disminuir las rigurosidades climáticas en espacios públicos.

Al hacer referencia a estrategias de diseño bioclimático se debe entender que no existen estrategias de acondicionamiento natural rígidas y únicas, las mismas están directamente relacionadas con condiciones de cada lugar.

A su vez, el nivel de asoleamiento de una ciudad es uno de los parámetros más determinantes en la calidad de vida urbana (Cárdenas y Vásquez, 2015; García, 2012). La relación descrita se fundamenta en que el sol es imprescindible para la vida humana, es la base de todas las energías sobre la tierra. No obstante, a nivel social, su presencia afecta en el estado de ánimo de los habitantes, "el bienestar y la alegría son componentes relacionados con el sol y la calidad de vida" (García, 2012, p. 4). Asimismo, la Organización Mundial de la Salud declara que el sol aporta numerosos beneficios para la salud (Lucas, Repacholi \& Mcmichael, 2006). En consecuencia, en la búsqueda de mejoras en la calidad urbana garantizar niveles óptimos de asoleamiento es uno de los principales objetivos. 
La importancia de contemplar el recurso solar en la planificación de ciudades más sostenibles es capital, ya que afecta en la calidad de vida de los habitantes tanto a nivel físico como anímico y resulta fundamental en la reducción del consumo energético de las urbes. Por tanto, es primordial, en términos de equidad, que todos los sectores habitables dispongan de la misma disposición del recurso o, al menos, de dos horas entre las $10: 00$ y $14: 00$ horas solares.

Las limitaciones de acceso al sol están dadas por las obstrucciones que los edificios circundantes puedan ejercer. En consecuencia, es necesario regular la relación entre la altura de las construcciones y las distancias entre las edificaciones colindantes y enfrentadas a ellas. La regulación de los niveles de asoleamiento de un asentamiento se desprende del estudio de la radiación solar y la distribución a lo largo del día. En entornos urbanos muy difícilmente se puede aprovechar la totalidad del recurso, consecuentemente, mediante el análisis de radiación y de las necesidades, tanto energéticas como psicológicas y/o biológicas, se establecen los niveles mínimos de acceso al sol (Cárdenas y Vásquez, 2015; García, 2012).

Este trabajo tiene por objetivo exponer una metodología de cálculo que permita determinar rangos de densidad poblacional en relación con las características de la trama urbana, la morfología edilicia y el clima del lugar, que garanticen el acceso al sol a la mayor cantidad de edificaciones.

\section{Metodología}

La metodología empleada para el desarrollo de este trabajo se estructura en dos etapas: primero se propone un procedimiento para el cálculo de valores de referencia para la densidad poblacional óptima en relación con las características de la trama urbana, al clima del lugar y al acceso al sol. En un segundo momento, la misma es aplicada en el Área Metropolitana de Mendoza (AMM), Argentina. Considerándose como óptimo un acceso al sol superior o igual a tres horas de potencial ganancia de la granja central (de 10:00 a 14:00 horas solares).

Con el fin de recabar la información necesaria para el cálculo de la densidad de habitantes sostenible de una ciudad respecto de los parámetros especificados de acceso al sol. En primer lugar, se deben estudiar las características del tejido urbano, identificando las particularidades de las distintas áreas de la urbe. A tal fin, es preciso saber el tamaño de las manzanas y el ancho de calles. Posteriormente, es necesario reconocer las características climáticas del sector (temperaturas máximas, medias y mínimas, la trayectoria solar y la radiación solar, dirección y la frecuencia de los vientos, humedad), para establecer lineamientos de acondicionamiento y estrategias urbanas a nivel bioclimático a fomentar.

Sobre la base de los datos climatológicos, se determinan los requerimientos particulares a nivel de morfología urbana o edilicia que promuevan el menor impacto ambiental de la ciudad en el terreno y se establecen las estrategias bioclimáticas que se fomentarán tanto nivel urbano como edilicio.

Una vez determinados los requerimientos se procede a definir el volumen edificable óptimo para cada sector de la trama, mediante el estudio de prototipos de manzana. Las fases de trabajo propuestas para el logro de dicho objetivo se desglosan a continuación.

\section{Determinación de alturas máximas}

Una vez determinados los requerimientos bioclimáticos se procede a determinar las alturas máximas edificables en relación con el acceso al sol. Para ello se recomienda la utilización de programas o técnicas que permitan evaluarlo. En este caso en particular se empleará el programa Heliodón ${ }^{R}$ desarrollado por Benoit Beckers y Luc Masset en 2004.

Para el cálculo de las alturas máximas se recomienda que la misma sea valorada en relación con los niveles o pisos, tomando como módulo para cada uno el valor de tres metros. Por lo que si la geometría solar nos permite una elevación de 12 metros, se considera que pueden ser cuatro niveles, o bien, si la misma establece 10 metros se computará como nueve metros. 


\section{Determinación de volumen teórico o superficies construidas máximas posibles en zonas con manzanero tradicional}

Para el cálculo de superficie máxima construida se requiere de la identificación de las distintas formas de manzanas y de la tipología edilicia más eficiente, en relación con los requerimientos de acondicionamiento bioclimático para la zona, establecidas en estudios particulares o previos. Una vez determinados todos los casos posibles se define el volumen edificable óptimo para cada uno, mediante el estudio de un prototipo de manzana.

Se recomienda, en un primer momento, establecer la huella edificada a nivel de manzana para posteriormente calcular la superficie construida en relación con los niveles (altura) máximos admisibles. Una vez determinada el área máxima edificable se establecen los indicadores Fracción de Ocupación del Suelo ${ }^{1}$ (FOS) y Fracción de Ocupación Total $^{2}$ (FOT) para cada modelo de manzana.

\section{Cálculo de la densidad de población óptima en relación con la trama urbana y el acceso al sol}

Para el cálculo de la población admisible se debe considerar la cantidad de metros cuadrados edificados por habitante. Una vez determinada la relación existente de construcción por habitante y sobre la base de los resultados obtenidos en el punto anterior se estipula la cantidad de habitantes máxima que podría albergar la misma, manteniendo los niveles actuales de construcción por habitante.

$$
H p=\frac{\sum \text { Sup. parcelaria } * \text { FOT }}{m 2 \text { construido por habitante }}
$$

Cantidad de habitantes zona parceladas (Hp)

Una vez determinada la cantidad de habitantes de los parcelarios del damero se procede a calcular la densidad poblacional de estos sectores.

Posteriormente, para la estimación de la población admisible de las superficies parcelarias o vacíos urbanos cuya forma interrumpe el damero tradicional, se realiza

\footnotetext{
${ }^{1}$ Factor de Ocupación del Suelo (FOS): relación entre la superficie construida en planta baja y la superficie del terreno.

${ }^{2}$ Factor de Ocupación Total (FOT): relación entre la superficie total construida y la superficie del terreno.
}

el cálculo definiendo cuántos habitantes tendría que tener la zona para obtener una densidad poblacional igual a la media de la esperada en las zonas con damero.

Densidad poblacional (hab/ha)

$$
=\frac{\text { Cantidad de hab.esperada de la fracción }}{\text { Sup.de la francción }}
$$

Población esperada $=\sum$ Densidad media $*$ Sup. del sector $(h a)$

\section{Desarrollo}

El AMM es un conglomerado urbano conformado por seis unidades político-administrativas contiguas en el territorio: Ciudad de Mendoza, Godoy Cruz, Guaymallén, Las Heras, Luján de Cuyo y Maipú (Figura 1). La misma se encuentra enclavada al pie de la Cordillera de los Andes y a más de 1.000 km del océano Atlántico; estas condiciones físicas demarcan la escasez de precipitaciones, dando como resultado un clima mayormente árido. La clasificación bioambiental, del conglomerado urbano según la normativa Argentina IRAM 11.603 es templado frío de montaña.
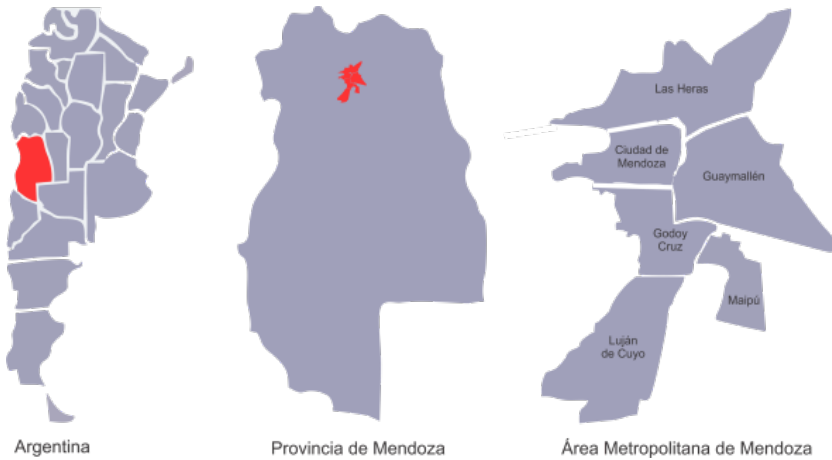

Figura 1: Ubicación y conformación del Área Metropolitana de Mendoza.

Fuente: elaboración propia.

La ciudad se caracteriza por tener inviernos fríos y veranos cálidos, con cielos claros durante todo el año. En la época invernal en el llano existen escasas precipitaciones níveas. Las temperaturas medias en verano rondan los $24^{\circ} \mathrm{C}$ y las de inviernos los $8^{\circ} \mathrm{C}$. La amplitud térmica entre las medias mínimas y máximas 
varían entre los $16^{\circ}$ y $17^{\circ} \mathrm{C}$ a lo largo de todo el año, lo cual responde a las condiciones de aridez de la zona.

Los requerimientos térmicos para una zona de confort entre los $18^{\circ}$ y $26^{\circ} \mathrm{C}$ denotan que las necesidades de calefacción superan a las de refrigeración. Las horas por debajo de los $18^{\circ} \mathrm{C}$ representan el $70,14 \%$ de las horas anuales, mientras que las horas de confort son del $21,5 \%$ y las que requieren de enfriamiento apenas el 8,33\% (Arboit y De Rosa, 2013; Mesa, 2003; Mesa y De Rosa, 2005).

La gran distancia al océano Atlántico y la barrera montañosa que se alza en dirección al Pacífico impiden la influencia marítima, generando que la región posea valores de nubosidad inferiores al 40\% (Mesa, 2003). Consecuentemente, la región cuenta con un alto recurso solar de entre 16,5 a $20 \mathrm{Mj} / \mathrm{m}^{2}$ día (Mesa, 2003), siendo en el período de verano cuando se dan los valores máximos mensuales.

El AMM se encuentra ubicada a los $32^{\circ} 41^{\prime}$ latitud sur, por lo que la posición del sol más desfavorable es la del 21 de junio, coincidente con los periodos más fríos. La inclinación solar para el solsticio de invierno al mediodía es de $34^{\circ}$. La mayor concentración de radiación se encuentra en las cuatro horas centrales del día, esto es, entre las $10: 00$ y las 14:00, con un ángulo de incidencia de $27^{\circ}$. La mayor incidencia solar es $81^{\circ}$ en el día 21 de diciembre, en el solsticio de verano.

El valor medio anual de Radiación Global sobre superficie horizontal es de $18.08 \mathrm{MJ} / \mathrm{m}^{2}$ por día y la media de Radiación Difusa sobre superficie horizontal $7.78 \mathrm{MJ} / \mathrm{m}^{2}$ por día. Los valores de radiación global son altos, presentando variaciones estacionales. El análisis de los valores dividido por la posición relativa en horizontal y verticales este, norte, sur denota que en el periodo con más requerimientos de calefacción la mayor captación se produce en la fachada norte, seguido por los techos. En verano la mayor radiación es recibida por las superficies horizontales, seguida por las este y oeste (Mesa, 2003). La radiación solar es intensa, lo que posibilita utilizar el sistema pasivo de Ganancia Solar Directa para calefacción, así como también los sistemas solares activos. Mientras que para el verano es necesario proyectar sistemas de protección solar fijos, móviles o vegetales.
Las características climáticas del AMM propician al máximo la implementación de estrategias de climatización que beneficien la utilización del recurso solar (Arboit y De Rosa, 2013). En los edificios bien diseñados, construidos y utilizados, es posible obtener hasta un $80 \%$ de la energía necesaria para calefacción (Arboit, Diblasi, Fernández Llano \& De Rosa, 2008).

En los meses donde las temperaturas superan los parámetros de confort, las bajas humedades relativas y la amplitud térmica entre el día y la noche posibilitan la implementación de estrategias de refrigeración, tanto de ventilación como de enfriamiento evaporativo. Las estrategias de refrigeración deben evitar la acumulación de calor mediante la protección de la radiación solar.

En el AMM, la forma de las manzanas con mayor potencial solar son las de formas rectangulares orientadas al norte (Figura 2), las cuales pierden potencial si se encuentran desviadas en más de $60^{\circ}$ respecto del Ecuador, mientras que las manzanas de formas más cuadradas tiene menor potencial colector, pero son más estables si su orientación varía (Mesa y De Rosas, 2001).

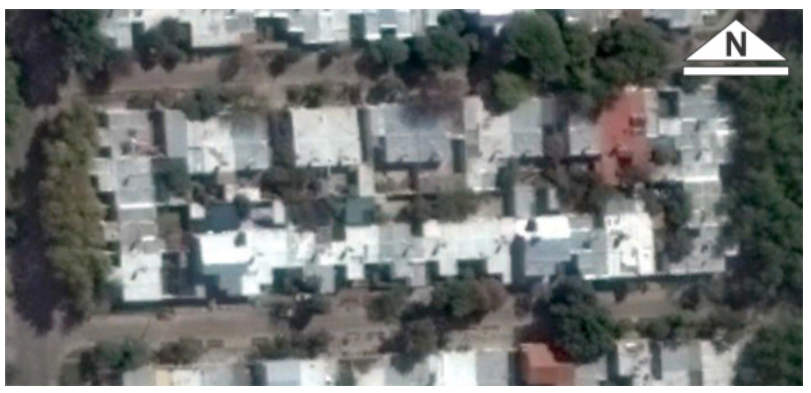

Figura 2: Ejemplo de manzana con mayor potencial solar. Fuente: Google Earth Pro.

Fuente: elaboración propia.

Distintas investigaciones realizadas en el área concuerdan que el comportamiento energético de las edificaciones se encuentra en relación a la forma de las mismas. Las formas compactas favorecen la conservación de energía, tanto aprovechadas de forma natural como la producida por artefactos de climatización. Del mismo modo mientras las volumetrías permiten mayor densidad poblacional, los consumos per-cápita son menores (Mesa, 2003). 
Desde el punto de vista de la forma del edificio, son convenientes formas simples con máximo desarrollo de la fachada $\mathrm{N}$ y mínimo de las $\mathrm{E}$ y $\mathrm{O}$. La estrategia primera debe ser la disminución de la superficie de envolvente, minimizando los intercambios de temperatura (Mesa, 2003).

La superficie urbanizada del AMM presenta una trama geométrica rectangular a rasgos generales, conformado por un $36 \%$ de circulaciones, $4 \%$ de espacios públicos de recreación (plazas, parques y peatonales) y un $60 \%$ de parcelas urbanas. La traza en cuadrícula es su principal característica morfológica, aunque no presenta las mismas características en toda su extensión. La misma, está compuesta por tres tipos de parcelarios, por un lado, está la presencia de fraccionamientos tradicionales inscriptos en manzanas tradicionales que acompañan la continuidad de las vías de circulación, por otro lado, se encuentran loteos realizados por privados que no presentan calles públicas ni con continuidad y por último terrenos agrarios o vacíos urbanos insertos en su totalidad en la trama consolidada. El manzanero tradicional representa el $45 \%$, mientras que los otros ascienden al 55\% (Gómez Piovano y Mesa, 2013). En el primer grupo existe variabilidad de dimensiones, las mismas alternan sus anchos entre los $20 \mathrm{~m}$ y los $120 \mathrm{~m}$, siendo las más representativas las de $40 \mathrm{~m}$ de ancho con una frecuencia del $28 \%$, seguida por las de $50 \mathrm{~m}$ con $18 \%$ y las de $100 \mathrm{~m}$ con un 14\% (Figura 3).

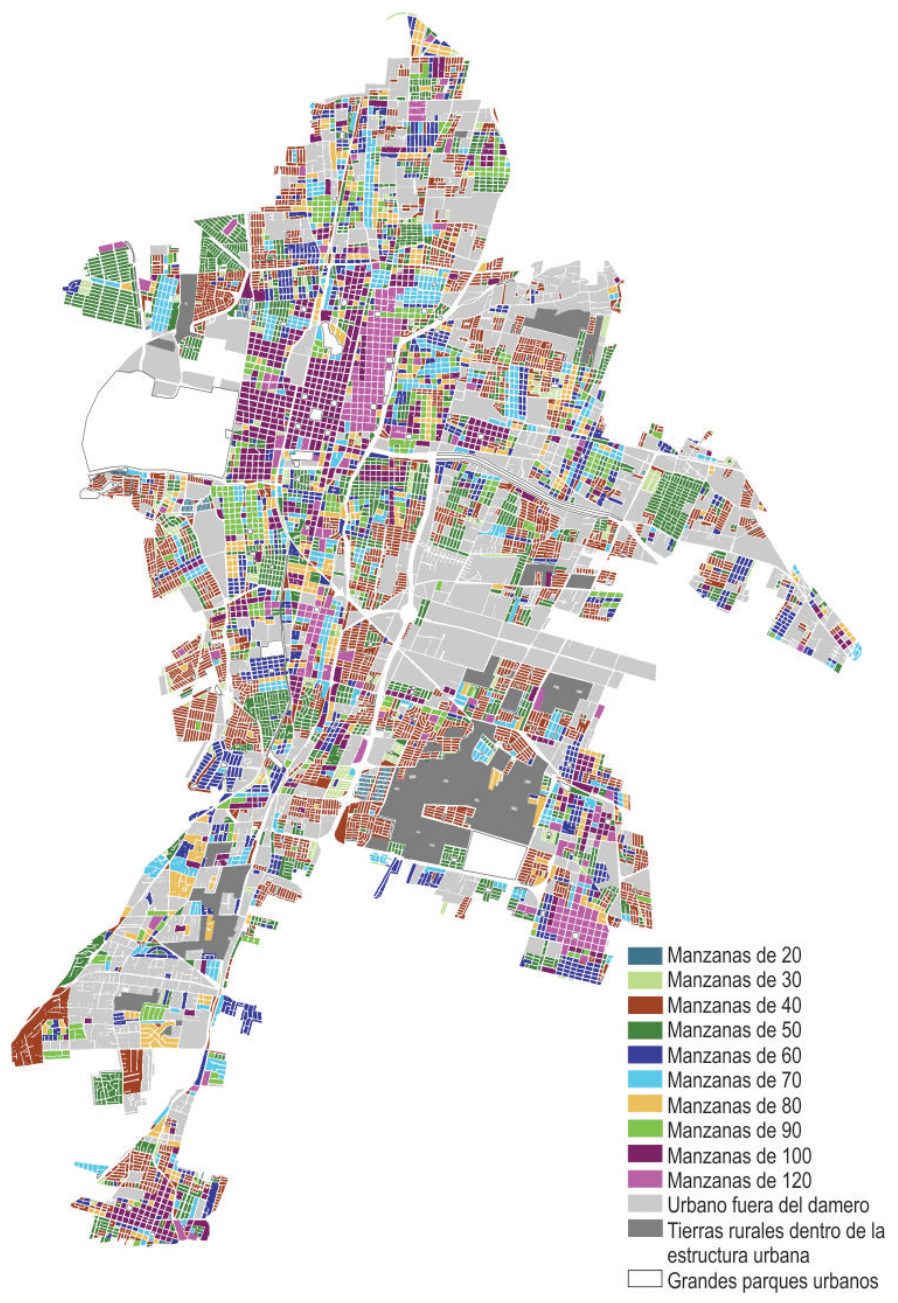

Figura 3: Composición de la trama urbana s/ ancho de manzana. Fuente: Elaboración propia. 
La relación actual entre superficie cubierta construida y la cantidad de habitantes es de $49,35 \mathrm{~m}^{2}$ por habitante, la cual varía dependiendo de las características de las distintas zonas. En los sectores más vulnerables o con menor poder adquisitivo la relación llega a ser de $10 \mathrm{~m}^{2}$ por habitante. La proporción más baja se relaciona tanto con la presencia de mayor cantidad de habitantes por unidades construidas como con la falta de equipamientos y servicios comerciales. Los sectores con mayor relación de superficie cubierta por habitante se corresponden con las áreas centrales o con nodos comerciales, administrativos y educacionales.

El FOT medio del AMM es del 0,70, con variaciones dependiendo del ancho de manzana. Los mayores índices se dan en las manzanas de $100 \mathrm{~m}$ de ancho con una media de 1,35, mientras que los menores valores corresponden a las manzanas de $40 \mathrm{~m}$ con un promedio de 0,54 .

La densidad habitacional media del AMM es de 53,52 habitantes por hectárea. La mayor concentración de personas se encuentra en los límites del departamento de Godoy Cruz, sector conformado por barrios en los cuales se radicaron los pobladores de villas de emergencia, donde la densidad del sector asciende a 114 habitantes por hectárea. Situación similar se produce en el sector oeste del mismo Departamento, donde radican 94 hab./ha. En el área considerada microcentro, ubicada en la parte este del Departamento Ciudad de Mendoza, la cantidad de personas es de 92 por superficie de medida. Los sectores menos poblados se encuentran en la periferia y poseen una relación de 9 u 11 hab./ha.

\section{Determinación de alturas máximas}

Como se expuso anteriormente el clima del sector requiere de la implementación de estrategias de climatización para calefacción que favorezcan la utilización del recurso solar y las formas edilicias que mejor comportamiento registran son las compactas (Mesa, 2003). Consecuentemente, la propuesta de morfología edilicia estable como principio la implementación de construcciones de fachada continua que favorezcan el acceso al recurso solar en las fachadas verticales y horizontales.

Con el objetivo de establecer las alturas máximas edificables para las diversas zonas del AMM, se realiza un estudio de la altura máxima que deben tener las edificaciones para garantizar el acceso solar de las construcciones adyacentes.

Para ello, se simulan volumetrías con distintas alturas (estableciéndose $3 \mathrm{~m}$ por piso de edificación) en el programa Heliodón ${ }^{R}$, tomando como parámetro el aseguramiento de la mayor cantidad de horas de ganancia solar para el día 21 de junio, solsticio de invierno, en el periodo de tiempo transcurrido entre las $10: 00$ y las $14: 00$ horas, para todas las fachadas verticales potencialmente colectoras, dado que en dicho periodo se presenta el mayor potencial de radiación.

El análisis realizado en el presente estudio denota que los distintos anchos de los canales viales pueden ser divididos en cuatro grupos. El primero incluye a todas aquellas calles cuyos anchos no superan los $10 \mathrm{~m}$, las edificaciones adyacentes a ella no deben ser mayores a un nivel ( $3 \mathrm{~m}$ de altura). En el segundo conjunto las calles tienen un ancho entre 10 y $13 \mathrm{~m}$, la altura máxima de las edificaciones que la rodean no puede exceder de los dos niveles o los $6 \mathrm{~m}$. El tercer grupo incluye a los canales viales que varían entre los 15 y los $19 \mathrm{~m}$ de amplitud, los mismos pueden contener construcciones de hasta tres niveles (9 m). El último conjunto está conformado por las vías de dimensiones mayores a $19 \mathrm{~m}$, donde en las inmediaciones las edificaciones pueden tener una altura máxima de cuarto niveles o 12 m (Figura 4 y 5). 


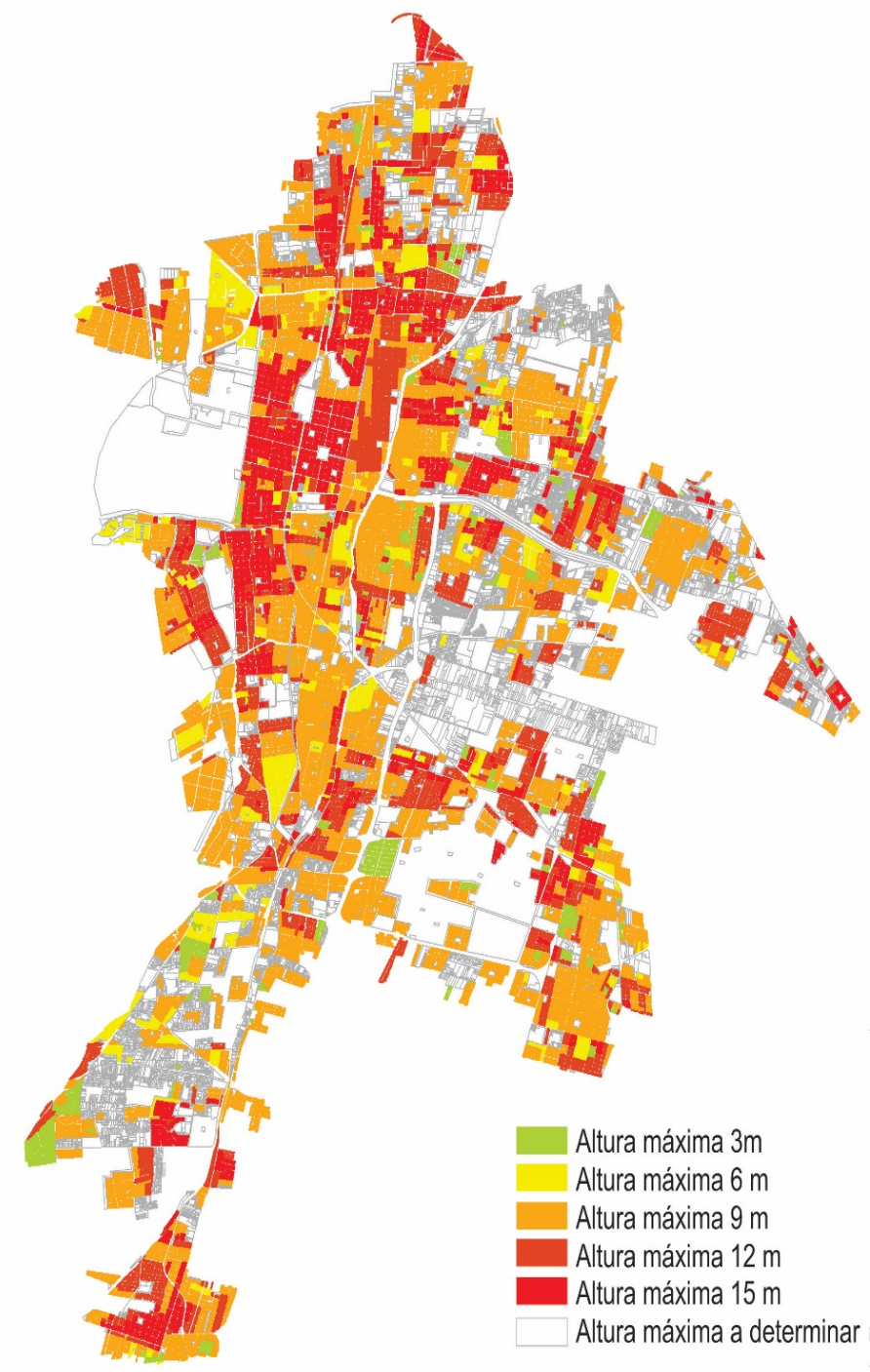

Figura 4: Alturas máximas propuestas para cada manzana del AMM.

Fuente: Elaboración propia.

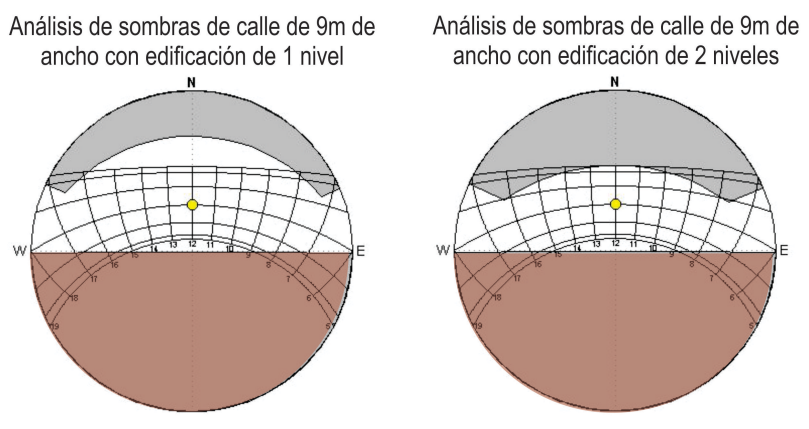

Análisis de sombras de calle de $14 \mathrm{~m}$ de ancho con edificación de 2 niveles

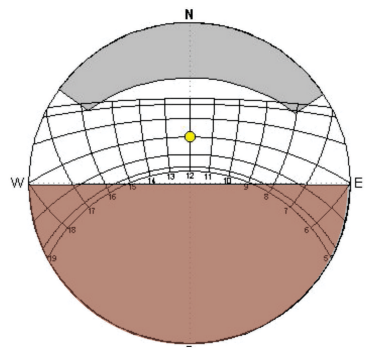

Análisis de sombras de calle de $19 \mathrm{~m}$ de ancho con edificación de 3 niveles

Análisis de sombras de calle de $14 \mathrm{~m}$ de ancho con edificación de 3 niveles

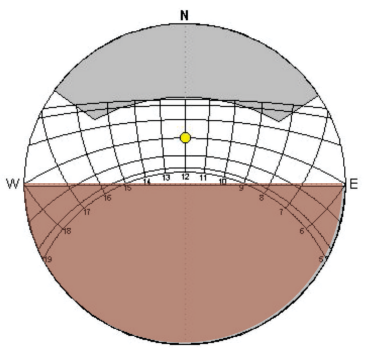

Análisis de sombras de calle de $19 \mathrm{~m}$ de ancho con edificación de 4 niveles
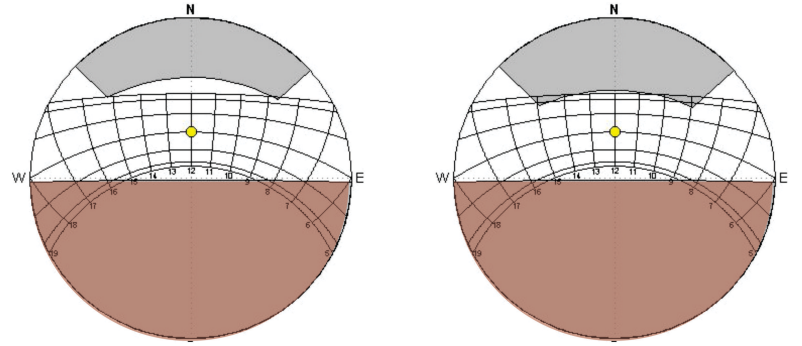

Sombras arrojadas por la edificación ubicada en la vereda de enfrente sobre la fachada Norte Sombras arrojadas por propia edificación sobre la fachada Norte

Figura 5: Análisis de sombras de distintas alturas de edificaciones en calles de diversos anchos. Fuente: Elaboración propia. 
Determinación de superficies construidas máximas posibles en zonas con manzanero tradicional

La estimación de las superficies máximas construibles se realiza en dos etapas distintas, primero se estipula la superficie máxima construida para las zonas urbanas con manzanero tradicional para, posteriormente, basándose en los resultados obtenidos se establece el área edificable de aquellos sectores que no presentan continuidad de la trama y de los terrenos agrarios o vacíos urbanos insertos en la trama consolidada.

Una vez determinadas las alturas máximas para cada ancho de calle se identifica la altura máxima para cada manzana. Se observa que en algunos casos las manzanas se encuentran rodeadas por canales viales de distintos tamaños; en estas situaciones se determina que toda la manzana - tanto en el perímetro como en el interiortendrá como altura máxima la correspondiente a la calle más estrecha.

Determinados los distintos grupos, se procede a establecer valores a la separación entre volúmenes construidos en el interior de la manzana, la que será igual o mayor al ancho de la calle y nunca menor a $7 \mathrm{~m}$ (Figura 6). El valor mínimo establecido se estable en relación con los estudios realizados sobre la incidencia de los corazones de manzanas en la mitigación de la isla de calor urbana del AMM realizados por Alicia Cantón (Cantón, Fernández, Mesa \& De Rosa, 2006).

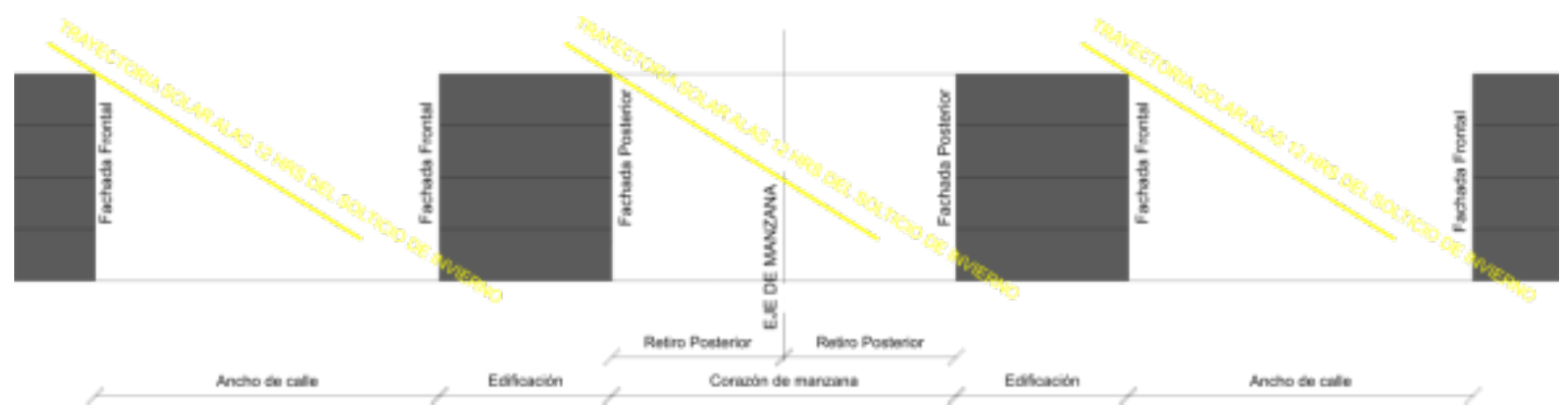

Figura 6: Corte de manzana tipo con trayectoria solar y retiros.

Fuente: Elaboración propia. 
Las edificaciones no podrán tener una distancia interior entre la fachada frontal y la posterior mayor a 18 $\mathrm{m}$, ya que del análisis que surge del catastro actual se considera que longitudes mayores requieren de patios internos, no garantizando la ventilación adecuada para los ambientes principales de la edificación.

En las manzanas cuyas dimensiones posibiliten la inclusión de más de un bloque edificado se estable que el retiro entre ambos es igual al ancho de calle determinado para el sector. Una vez establecidas las superficies de terreno edificables se calcula el valor del FOS; para determinar el FOT se relaciona el primer indicador con la altura máxima edificable (Figura 7).

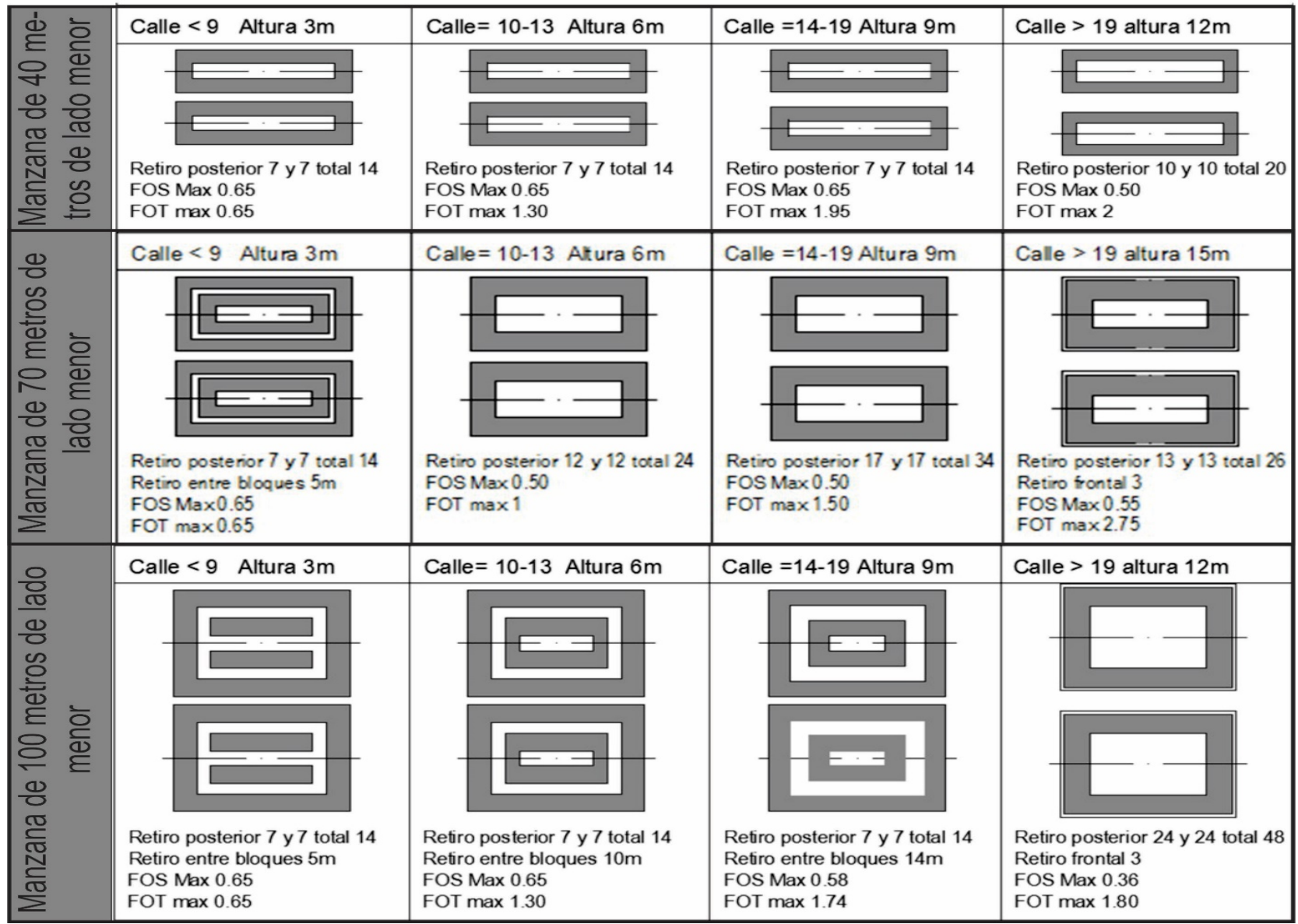

Figura 7: Valores de FOT propuestos para manzanas de distintas dimensiones y anchos de calle del AMM.

Fuente: Elaboración propia. 


\section{Cálculo de la densidad de población óptima en relación} con la trama urbana y el acceso al sol

Una vez determinados los valores de FOS Y FOT para cada tipología de manzanero se procede a calcular el área edificada máxima. Para el caso del AMM la misma para las parcelas tradicionales es de $122.731 .020 \mathrm{~m}^{2}$. Tomando como base que la relación de superficie construida por habitante se mantendría en los niveles actuales de $50 \mathrm{~m}^{2}$, la capacidad construida de los parcelarios tradicionales implicaría una población de 2.454.620 personas.

El análisis realizado en el presente estudio denota que la población no se distribuye del mismo modo en todo el territorio, ya que la variación de la capacidad constructiva para los distintos sectores del AMM determina densidades poblacionales que van desde 69 hasta los 400 hab./ha (Figura 8 ).

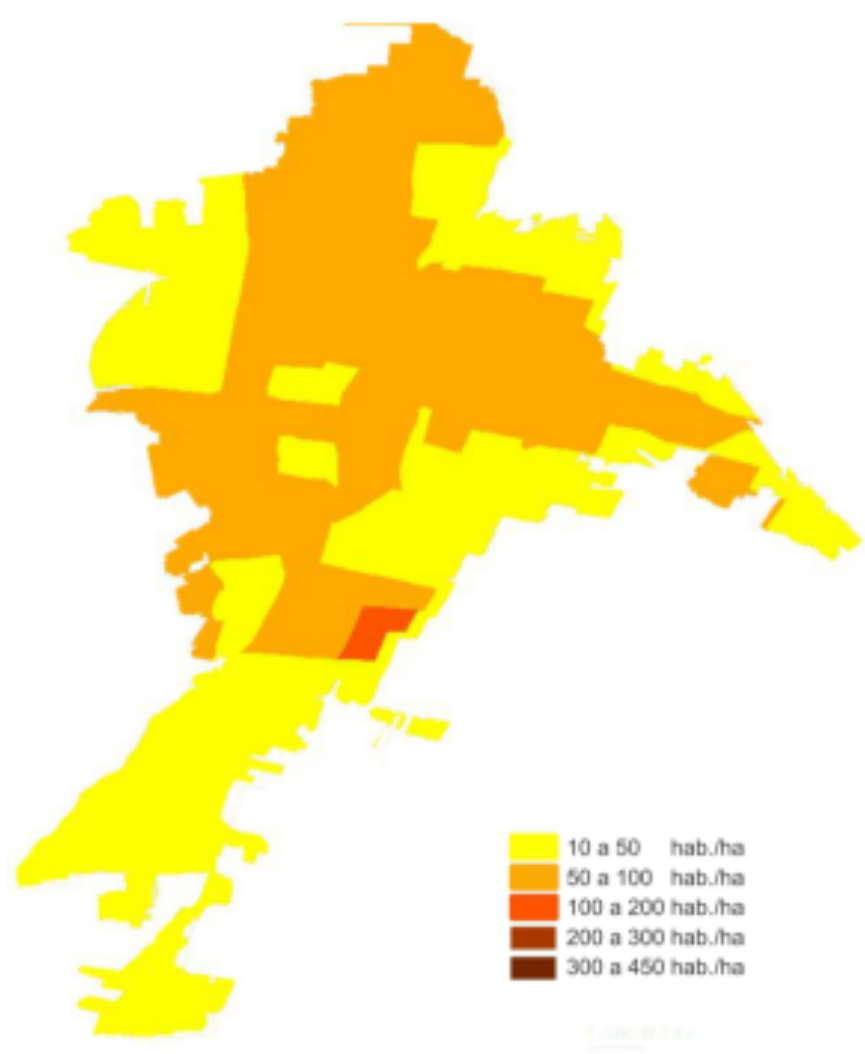

\section{Densidad Población Actual}

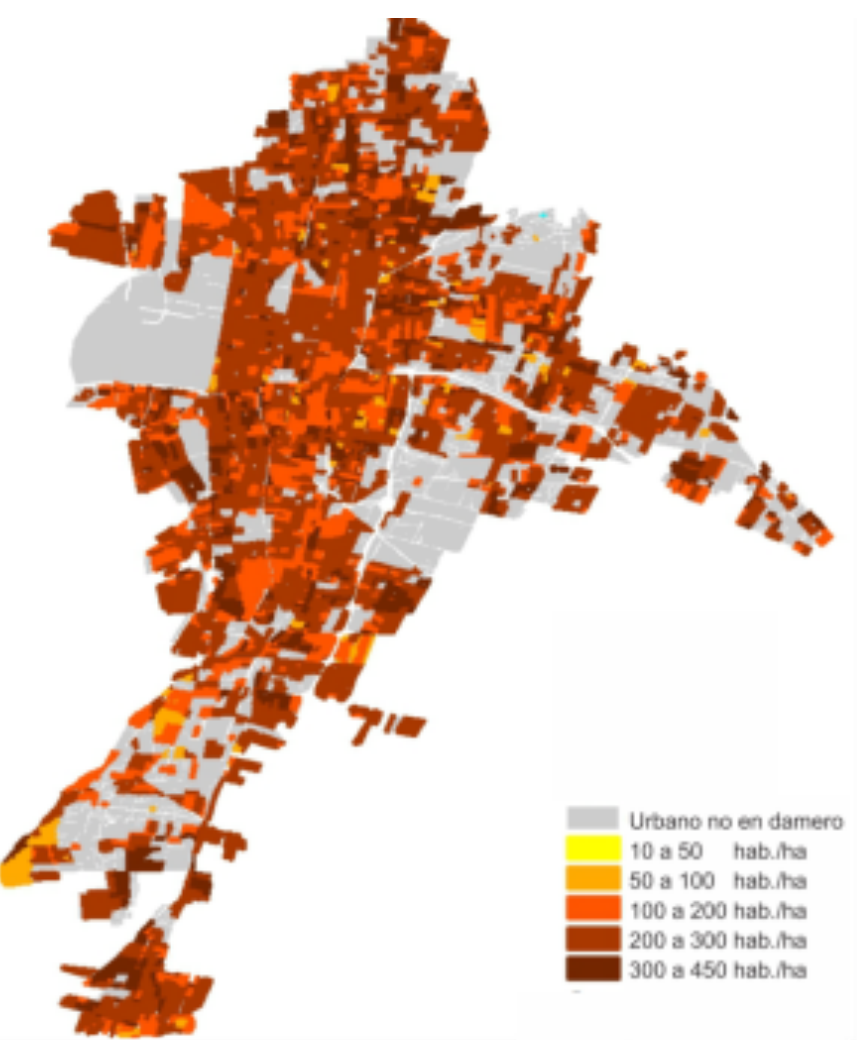

Densidad Población Posible en relación al acceso al sol

Figura 8: Densidades poblacionales actuales y densidades máximas propuestas para las distintas zonas del AMM.

Fuente: Elaboración propia. 


\section{Conclusiones}

Las características climáticas del AMM permiten la implementación de sistemas que aprovechen la disponibilidad del recurso solar, puesto que la zona posee grandes potencialidades para el acondicionamiento térmico natural de las edificaciones. Los edificios que emplean sistemas de acondicionamiento natural en el sector tienen ahorros energéticos de hasta el $80 \%$. Es por ello que no asegurar la factibilidad de implementación de estrategias de climatización natural de los espacios interiores atenta contra los principios de eficiencia energética y sustentabilidad urbana.

Por otra parte, garantizar ganancia solar para todas las edificaciones en el periodo de invierno es una limitante a la capacidad constructiva y, por consiguiente, a la densidad poblacional. En las calles angostas y manzanas pequeñas los volúmenes edificables son menores que en las calles más amplias.

Otro aspecto del urbanismo bioclimático que impacta en la morfología urbana es el comportamiento térmico de las edificaciones. En efecto, en zonas como las del AMM disminuir las superficies expuestas es fundamental para un acondicionamiento eficiente. Consecuentemente, las edificaciones de fachada continua son las que mejor desempeño poseen en tipologías de baja altura como las que suscitan el ancho de calles del sector.

El estudio de la capacidad máxima constructiva en relación con el acceso al sol del AMM revela que no se deben proyectar densidades habitacionales generales para toda la ciudad si el objetivo es mejorar la sustentabilidad urbana. Por el contrario, las mismas deben proyectarse en relación con las particularidades del tejido urbano de cada sector. Por ejemplo, en sectores con manzanas de $40 \mathrm{~m}$ de ancho y calles de amplitud menor a $10 \mathrm{~m}$ las densidades observadas se encuentran en 80 hab./ha, mientras que la misma dimensión de manzana, pero con vías mayores a $19 \mathrm{~m}$ la densidad máxima es de 395 hab./ha.

De acuerdo con lo expuesto, los valores de los indicadores urbanos de densidad poblacional planteados como sustentables en distintos estudios (Higueras, 2009; Marín, 2012; Rueda, 2011) no son aplicables en ciudades como Mendoza. Los sistemas unificadores de las densidades constructivas no son convenientes de aplicar en tramas donde los tamaños de manzanas y los anchos de calles son variables. En sectores con cuadras angostas y calles estrechas la densidad debe ser menor.

En conclusión, la incorporación de valores de referencia de indicadores urbanos establecidos para otras ciudades sin una previa evaluación puede derivar en modelos que degraden el ambiente construido en vez de mejorarlo. La estandarización de niveles de densidad poblacional sin la consideración de las características particulares de cada sector resulta contraproducente, por lo que deben generarse metodologías que permitan establecer estrategias y valores de densidad para cada urbe.

Arboit, M., Diblasi, A., Fernández Llano, J. C., \& De Rosa C. (2008). Assessing the solar potential of low density urban environments in Andean Cities with desert climates - The case of the city of Mendoza, in Argentina. Renewable Energy, 33(8), 1733-1748. http://dx.doi.org/10.1016/j.renene.2007.11.007

Cantón, M. A., Fernández, J., Mesa, A., \& De Rosa, C. (2006). Energy assessment of the Patio-House Evolution. Trabajo presentado en la International Conference "Living in hot Deserts: Is a sustainable

Arboit, M. y De Rosa, C. (2013). Estrategias para la sostenibilidad energética del sector edilicio urbano en zonas de climas secos. Evaluación comparativa de la actualización del Código Urbano y de Edificación de la Ciudad de Mendoza, Argentina. Revista de Urbanismo, 28, 94-113. Recuperado de http://www.revistaurbanismo.uchile.cl/index.php/RU /article/viewPDFInterstitial/21561/30058 
urban design still possible in arid regions", Ghardaïa, Algeria.

Cárdenas, L. A. y Vásquez, J. P. (2015). Potencial solar en fachadas integrando la densidad urbana. Una mirada crítica a la norma urbanística chilena. AUS, 18, 58-63. https://doi.org/10.4206/aus.2015.n18-10

Cárdenas, L. A. y Uribe, P. (2012). Acceso solar a las edificaciones. El eslabón pendiente en la legislación urbanística chilena sobre la actividad proyectual. Revista de Urbanismo, 26, 21-42. https://doi.org/10.5354/0717-5051.2012.20922

Cepeda, M. y Mardaras, I. (2004). Cuantificación energética de la construcción de edificios y el proceso de urbanización. ConArquitectura: arquitectura con arcilla cocida, 12, 65-80. Recuperado de https://dialnet.unirioja.es/servlet/revista?codigo=8887

Cummins, S. K. \& Jackson, R. J. (2001). The built environment and children's health. Pediatric Clinics of North America, 48(5), 1241-1252. Recuperado de https://doi.org/10.1016/s0031-3955(05)70372-2

Gaja, F. (2008) Urbanismo Ecológico, ¿sueño o pesadilla? Sostenibilidad, tecnología y humanismo, 3, 105-157. Recuperado de https://upcommons.upc.edu/bitstream/handle/2099/ 7079/gaja.pdf

García, F. (2012). Densificación con criterios de soleamiento. Un método gráfico para el cálculo de áreas adecuadas para nueva edificación en entornos construidos en el caso de Zabalgana en VitoriaGasteiz. Trabajo presentado en el Congreso Nacional del Medio Ambiente, Madrid, España.

Gómez Piovano, J. y Mesa, A. (2013) Estudio de las implicancias de aplicación de valores de referencia para indicadores urbanos desarrollados en Europa en ciudades de América Latina, y elaboración de una metodología de cálculo. Avances en Energías Renovables y Medio Ambiente, 17(1), 11-20. Recuperado de http://www.cricyt.edu.ar/asades/averma.php

González, M. (2005). Indicadores básicos para la planificación de la sostenibilidad urbana local. Biblio $3 W$, Revista bibliográfica de geografía y ciencias sociales, 10(586). Recuperado de http://www.ub.edu/geocrit/b3w-586.htm
Hernández, A. (2009). Calidad de vida y medioambiente urbano. Indicadores locales de sostenibilidad y calidad de vida urbana. Revista INVI, 65(24), 79-111. http://dx.doi.org/10.4067/S071883582009000100003

Higueras, E. (2009). El reto de la ciudad habitable y sostenible. Madrid: DAPP.

Lopez, L. y Relea, C. (2002). Ciudades y periferias sostenibles. Una revisión de conceptos y enfoques aplicados a España y Castilla y León. Polígonos. Revista de Geografía, 11-12, 113-136. Recuperado de: http://revpubli.unileon.es/index.php/poligonos/articl e/viewFile/523/464

Lucas, R., Repacholi, M., \& Mcmichael, A. (2006). Is the current public health message on UV exposure correct? Bulletin of the World Health Organization, 84(6), 485-491. https://dx.doi.org/10.1590/S004296862006000600018

Marín, P. (2012). Modelos urbanos sostenibles. Málaga: Servicio de Programas del Ayuntamiento de Málaga Observatorio de Medio Ambiente Urbano.

Mesa, A. y De Rosas, C. (2001). La incidencia de las variables morfológicas sobre la eficiencia energética de la edilicia urbana en el Área Metropolitana de Mendoza. Avances en Energías Renovables y Medio Ambiente, 5(7), 37-42. Recuperado de http://www.cricyt.edu.ar/asades/averma.php

Mesa, A. (2003). Método teórico de diagnóstico de la habitabilidad termo-lumínica del espacio arquitectónico, como base para la planificación urbana: Caso Mendoza, Argentina (Tesis inédita de doctorado). Universidad de Mendoza, Mendoza.

Mesa, A. y De Rosa, C. (2005). Estudio de los patrones de apropiación del suelo urbano por la expansión de las áreas residenciales. Análisis del Área Metropolitana de Mendoza. Avances en Energías Renovables y Medio Ambiente, 9(05) 05, 91-96. Recuperado de http://www.cricyt.edu.ar/asades/averma.php

Norman, J., Maclean, H., Asce, M., \& Kennedy, C. (2006). Comparing high and low residential density: Life-cycle analysis of energy use and greenhouse gas emissions. Journal of Urban Planning and Development, 132(1), 10-21. http://dx.doi.org/10.1061/(ASCE)07339488(2006)132:1(10) 
Rueda, S. (2011). El urbanismo ecológico. Barcelona: Agencia d'Ecologia urbana de Barcelona.

Ruiz, M. A., Correa, E., y Cantón, M. A. (2012). Función ambiental de parques urbanos en zonas áridas: clima y confort térmico. Trabajo presentado en ENTAC 2012 - XIV Encontro Nacional de Tecnología do Ambiente Construido. Brasil, Associação Nacional de Tecnologiado Ambiente Construído.

Valenzuela, M. (2009) Ciudad y sostenibilidad el mayor reto urbano del siglo XXI. Lurralde. Investigación y espacio, 32, 405-436. Recuperado de http://www.uam.es/gruposinv/urbytur/documentos/ 32valenzuela.pdf 